\title{
Isomorphic Multiplicative Transitivity for Intuitionistic and Interval-valued Fuzzy Preference Relations and Its Application in Deriving Their Priority Vectors
}

\author{
Jian Wu, Senior Member, IEEE, Francisco Chiclana, Senior Member, IEEE, and Huchang Liao, Member, IEEE
}

\begin{abstract}
Intuitionistic fuzzy preference relations (IFPRs) are used to deal with hesitation while interval-valued fuzzy preference relations (IVFPRs) are for uncertainty in multi-criteria decision making (MCDM). This article aims to explore the isomorphic multiplicative transitivity for IFPRs and IVFPRs, which builds the substantial relationship between hesitation and uncertainty in MCDM. To do that, the definition of the multiplicative transitivity property of IFPRs is established by combining the multiplication of intuitionistic fuzzy sets and Tanino's multiplicative transitivity property of fuzzy preference relations (FPRs). It is proved to be isomorphic to the multiplicative transitivity of IVFPRs derived via Zadeh's Extension Principle. The use of the multiplicative transitivity isomorphism is twofold: (1) to discover the substantial relationship between IFPRs and IVFPRs, which will bridge the gap between hesitation and uncertainty in MCDM problems; and (2) to strengthen the soundness of the multiplicative transitivity property of IFPRs and IVFPRs by supporting each other with two different reliable sources, respectively. Furthermore, based on the existing isomorphism, the concept of multiplicative consistency for IFPRs is defined through a strict mathematical process, and it is proved to satisfy the following several desirable properties: weak-transitivity, max-max-transitivity, and center-divisiontransitivity. A multiplicative consistency based multi-objective programming (MOP) model is investigated to derive the priority vector from an IFPR. This model has the advantage of not losing information as the priority vector representation coincides with that of the input information, which was not the case with existing methods where crisp priority vectors were derived as a consequence of modelling transitivity just for the intuitionistic membership function and not for the intuitionistic non-membership function. Finally, a numerical example concerning green supply selection is given to validate the efficiency and practicality of the proposed multiplicative consistency MOP model.
\end{abstract}

\section{Index Terms}

Intuitionistic; Interval-valued; fuzzy preference relations; Isomorphic multiplicative transitivity; Priority vector

\section{INTRODUCTION}

$\mathbf{I}$ $\mathrm{N}$ multi-criteria decision making (MCDM), experts usually need to compare a finite set of alternatives $X=\left\{x_{1}, x_{2}, \ldots, x_{n}\right\}$ with respect to a set of criteria, and construct preference relations (PRs). An appropriate information representation format widely used in these cases is the fuzzy preference relation (FPR) [1], i.e. a reciprocal [0,1]-valued PR: $P=\left(p_{i j}\right) ; \forall i, j: 0 \leq$ $p_{i j} \leq 1, p_{i j}+p_{j i}=1$. However, many decision making processes take place in an environment in which the information is not precisely known, and therefore experts may not be able to accurately express their preferences using a single crisp value in $[0,1]$. This could happen, for example, when experts are not confident about their judgments, i.e. there exists hesitation regarding their preferences [2], [3]. Atanassov's intuitionistic fuzzy sets (IFSs) [4] are based on the use of membership degree $(\mu)$, non-membership degree $(\nu)$ and hesitation index $(\tau \equiv 1-\mu-\nu)$ to model experts' subjective preferences, and therefore intuitionistic fuzzy preference relations (IFPRs) can be regarded as more appropriate than FPRs to deal with hesitation. Recently, the use of IFPRs in MCDM problems in fuzzy environments has attracted the attention of many researchers (see for example [5]-[10]).

One key issue in MCDM problems with IFPRs is how to correctly model the concept of consistency. One of the main advantages of using PRs is that of focusing exclusively on two alternatives at a time as this facilitates experts when expressing their preferences. However, it also limits experts' global perception of the alternatives and it generates more information than is really necessary, which ultimately could lead to the provision of conflicting information and therefore inconsistency may arise [11]. Recently, $\mathrm{Xu}$ et al. [12] introduced the concept of multiplicative consistency property for IFPRs and used it to estimate possible missing elements in an IFPR. However, this property as defined by Xu et al. [12] was in conflict with Taninos' multiplicative transitivity property for FPRs. Indeed, it is well known that IFPRs extend FPRs and therefore the multiplicative consistency property for IFPRs should derive in Tanino's multiplicative transitivity property when an IFPR becomes a FPR, i.e. the following $\mu_{i j} \mu_{j k} \mu_{k i}=\mu_{i k} \mu_{k j} \mu_{j i}, \forall i, k, j \in\{1,2, \ldots n\}$ should be verified. This condition, however, does not hold

Jian Wu is with the School of Economics and Management, Shanghai Maritime University, Shanghai 201306, China (jyajian@163.com). Francisco Chiclana is with the Centre for Computational Intelligence (CCI), Faculty of Technology, De Montfort University, Leicester, UK (chiclana@dmu.ac.uk). Huchang Liao is with the Business School, Sichuan University, Chengdu 610064, China (liaohuchang@163.com). 
using the definition by $\mathrm{Xu}$ et al. [12], which was later acknowledged by Liao and $\mathrm{Xu}$ in [13]. This same issue has also been addressed recently (see for example [14], [15]). Therefore, it is necessary to develop a theoretically sound framework for modelling consistency of IFPRs.

Consistency of preferences has traditionally been associated with the transitivity property [17]. Indeed, the concept of consistency in a crisp context has been defined in terms of acyclicity [18], which is closely related to the transitivity of the corresponding binary $\mathrm{PR}$, in the sense that if alternative $x_{i}$ is preferred to alternative $x_{j}$ and this one to alternative $x_{k}$ then alternative $x_{i}$ should be preferred to alternative $x_{k}$. In the case of FPRs, the traditional requirement to characterise consistency is to use properties that extend the classical requirements of binary PRs. The main difference in this case with respect to the binary one resides on the role the intensity of preference has, and as such a variety of implementations have resulted in different proposals to model consistency for FPRs. In any case, consistency of FPRs is also based on the notion of transitivity, and in particular the multiplicative transitivity property proposed by Tanino in [19] has been proved to be the most appropriate property for modelling cardinal consistency of FPRs in [11]. Recall that FPRs are particular cases of IFPRs, and then, the same conclusion can be applied to this later type of information. Thus, the first objective in this paper is to extend the formulation of multiplicative transitivity property from FPRs to IFPRs, using a strict mathematical methodology. The proposed multiplicative transitivity of IFPRs has the advantage of covering the proposed formulation for membership grades $(\mu)$ proposed by Liao and Xu in [13], but it also covers the non-membership grades $(\nu)$ with the following formulation: $\left(1-\nu_{i j}\right)\left(1-\nu_{j k}\right)\left(1-\nu_{k i}\right)=\left(1-\nu_{i k}\right)\left(1-\nu_{k j}\right)\left(1-\nu_{j i}\right)$. The use of the multiplicative transitivity property proposed in this paper implies that the priority vector derived from IFPRs will be of the same type to the original preferences, a claim that cannot be stated when using Liao and Xu's proposal [13]. Consequently, this paper proposes a multiplicative transitivity property that is deemed reasonable to model the consistency property of IFPRs.

Another interesting issue is to reveal the substantial relationship between IFPRs and IVFPRs. Apart from the hesitation, uncertainty may also be present: an expert may not possess a precise or sufficient level of knowledge of the problem to solve. This uncertainty is usually dealt with interval-valued fuzzy preference relations (IVFPRs), in which the elements are interval-valued fuzzy numbers (IVFNs) [20]-[25]. Recently, the relationship between hesitation and uncertainty has attracted some interest from researchers. The IFSs and interval-valued FSs (IVFSs) are mathematically equivalent [26], and IFPRs and IVFPRs have been proved to be mathematically isomorphic by $\mathrm{Wu}$ and Chiclana [27]. However, mathematical isomorphism is just the superficial relationship and it cannot guarantee the reasonability of converting processes between IFPRs and IVFPRs in a straightforward manner unless formal theoretically sound results are provided. Therefore, the second objective of this article is to find a substantial relationship (multiplicative transitivity isomorphism) between IFPRs and IVFPRs. To do that, the definition of multiplicative transitivity for IVFPRs based on Zadeh's Extension Principle [28] is introduced first. Then, the multiplicative transitivity isomorphism is established with a rigorous mathematical proof. Therefore, the reasonability for these two types of multiplicative transitivity for IFPRs and IVFPRs is strengthened by each other because they are set up via different reliable methods: Operational laws of IFSs and Extension Principle and Representation Theorem of Fuzzy Sets $(F S s)$, respectively. Thus, IFPRs and IVFPRs not only have mathematical isomorphism, but also have multiplicative transitivity isomorphism. Consequently, IFPRs and IVFPRs are completely equivalent in consistency based MCDM resolution processes, and then hesitation and uncertainty can be unified. Finally, a multiplicative transitivity based multi-objective programming (MOP) model for deriving the priority vector of IFPRs is proposed.

The rest of this article is set out as follows: In section II the multiplicative transitivity property for IFPRs is derived based on the corresponding property for FPRs and the multiplication operator of IFSs. The multiplicative transitivity isomorphism between IFPRs and IVFPRs is established in Section III, where the concept of multiplicative consistency for IVFPRs is derived via the corresponding property for FPRs and Zadeh's extension principle. A study of some of its properties is included in Section IV, which is followed by a multiplicative consistency based multi-objective programming (MOP) model to derive the associated priority vector. Section V includes an illustrative example to verify the efficiency of the proposed method. Finally, Section VI provides an analysis of the proposed multiplicative transitivity that highlights the main differences with respect to the existing models. The article ends with some concluding remarks in Section VII.

\section{Multiplicative Transitivity Property FOR IFPRs}

Atanassov introduced the concept of IFS in [4]:

Definition 1. Given a universe of discourse, $X$, an intuitionistic fuzzy set (IFS) $A=\left\{\left\langle x, \mu_{A}(x), \nu_{A}(x)\right\rangle \mid x \in X\right\}$ over $X$ is characterised by a membership function $\mu_{A}: X \rightarrow[0,1]$ and a non-membership function $\nu_{A}: X \rightarrow[0,1]$ verifying: $0 \leq \mu_{A}(x)+\nu_{A}(x) \leq 1 \quad \forall x \in X$

When $\mu_{A}(x)=1-\nu_{A}(x) \forall x \in X$, IFSs become fuzzy sets (FSs) and their reciprocity relationship makes the nonmembership function unnecessary as it can be derived from the membership function. If $\exists x \in X: \mu_{A}(x)<1-\nu_{A}(x)$ then the hesitancy function, $\tau_{A}: X \rightarrow[0,1]$, is defined with $\tau_{A}(x)=1-\mu_{A}(x)-\nu_{A}(x)$ interpreted as the hesitation margin of the membership of element $x$ to the IFS $A$, i.e. the amount of lacking information when determining the membership of $x$ to $A$ [29]. 
For computational convenience, $\mathrm{Xu}$ and Yager [30] introduced the definition of the intuitionistic fuzzy number (IFN) as: $A=\left\{\left\langle x,\left[\mu_{A}(x), 1-\nu_{A}(x)\right]\right\rangle \mid x \in X\right\}$. Given two IFNs $r_{i j}=\left[\mu_{i j}, 1-\nu_{i j}\right]$ and $r_{k l}=\left[\mu_{k l}, 1-\nu_{k l}\right]$, respectively, the following operators were introduced by $\mathrm{Xu}$ and Yager [30]:

- $r_{i j} \oplus r_{k l}=\left[\mu_{i j}+\mu_{k l}-\mu_{i j} \mu_{k l}, 1-\nu_{i j} \nu_{k l}\right]$

- $r_{i j} \otimes r_{k l}=\left[\mu_{i j} \mu_{k l},\left(1-\nu_{i j}\right)\left(1-\nu_{k l}\right)\right]$

- $\lambda r_{i j}=\left[1-\left(1-\mu_{i j}\right)^{\lambda}, 1-\nu_{i j}^{\lambda}\right], \lambda>0$

- $r_{i j}^{\lambda}=\left[\mu_{i j}^{\lambda},\left(1-\nu_{i j}\right)^{\lambda}\right], \lambda>0$

In a decision making context with three given alternatives $x_{i}, x_{j}, x_{k}$, the question whether the 'degree or strength of preference' of alternative $x_{i}$ over alternative $x_{j}$ exceeds, equals, or is less than the 'degree or strength of preference' of alternative $x_{j}$ over alternative $x_{k}$ when alternative $x_{i}$ is preferred to alternative $x_{j}$ and alternative $x_{j}$ to alternative $x_{k}$, cannot be answered by the classical preference modelling [11] but it can with the fuzzy preference modelling. The application of the concept of fuzzy set to a binary relation leads to the concept of a fuzzy preference relation [1]:

Definition 2. A fuzzy preference relation (FPR) $P$ on a finite set of alternatives $X=\left\{x_{1}, \ldots, x_{n}\right\}$ is characterised by a membership function $\mu_{P}: X \times X \longrightarrow[0,1], \mu_{P}\left(x_{i}, x_{j}\right)=p_{i j}$, verifying: $p_{i j}+p_{j i}=1, \forall i, j \in\{1, \ldots, n\}$.

In this context, a FPR is usually denoted by a matrix $P=\left(p_{i j}\right)$, with the following interpretation: (i) $p_{i j}=1$ indicates the maximum degree of preference for $x_{i}$ over $x_{j}$; (ii) $p_{i j} \in[0.5,1]$ indicates a definite preference for $x_{i}$ over $x_{j}$; (iii) $p_{i j}=0.5$ indicates indifference between $x_{i}$ and $x_{j}$. A FPR is also known as a reciprocal [0,1]-valued preference relation.

Considering that in some real-life situations experts may not be able to accurately express their preferences for alternatives due to a lack of precise knowledge of the problem, the use of intuitionistic preference values might be more appropriate than fuzzy preference ones. The intuitionistic FPR (IFPR) [31] extends the the concept of a FPR given in the above Definition 2:

Definition 3. An intuitionistic fuzzy preference relation (IFPR) $R$ on a finite set of alternatives $X=\left\{x_{1}, \ldots, x_{n}\right\}$ is characterised by a membership function $\mu_{R}: X \times X \rightarrow[0,1]$ and a non-membership function $\nu_{R}: X \times X \rightarrow[0,1]$ verifying $0 \leq \mu_{R}\left(x_{i}, x_{j}\right)+\nu_{R}\left(x_{i}, x_{j}\right) \leq 1 \quad \forall\left(x_{i}, x_{j}\right) \in X \times X$ and (i) $\mu_{i i}=\nu_{i i}=0.5 \forall i \in\{1, \ldots, n\} ;$ (ii) $\mu_{j i}=\nu_{i j} \forall i, j \in\{1, \ldots, n\}$. The value $\mu_{R}\left(x_{i}, x_{j}\right)=\mu_{i j}$ is interpreted as the certainty degree up to which $x_{i}$ is preferred to $x_{j}$, while the value $\nu_{R}\left(x_{i}, x_{j}\right)=\nu_{i j}$ represents the certainty degree up to which $x_{i}$ is non-preferred to $x_{j}$. Using matrix notation, an IFPR is represented as $R=\left(r_{i j}\right)=\left(\left\langle\mu_{i j}, \nu_{i j}\right\rangle\right)$.

In decision making problems with FPRs, several consistency properties are usually assumed to minimise the impact of inconsistent opinions in the decision process. For IFPRs, Xu et al. in [12] proposed the following multiplicative consistency property:

$$
\forall i<k<j, \mu_{i j}=\left\{\begin{array}{l}
0,\left(\mu_{i k}, \mu_{k j}\right) \in\{(0,1),(1,0)\} \\
\frac{\mu_{i k} \mu_{k j}}{\mu_{i k} \mu_{k j}+\left(1-\mu_{i k}\right)\left(1-\mu_{k j}\right)}, \text { otherwise }
\end{array}\right.
$$

and

$$
\forall i<k<j, \nu_{i j}=\left\{\begin{array}{l}
0,\left(\nu_{i k}, \nu_{k j}\right) \in\{(0,1),(1,0)\} \\
\frac{\nu_{i k} \nu_{k j}}{\nu_{i k} v_{k j}+\left(1-\nu_{i k}\right)\left(1-\nu_{k j}\right)}, \text { otherwise }
\end{array}\right.
$$

However, Liao and $\mathrm{Xu}$ in [13] pointed that this multiplicative consistency property is not correct as it does not derive in the multiplicative consistency property for FPRs (recall that FPRs are IFPRs with null hesitancy) that is regarded as the basic foundation of multiplicative transitivity for IFPRs: $\mu_{i j} \mu_{j k} \mu_{k i}=\mu_{i k} \mu_{k j} \mu_{j i}, \forall i, k, j$. However, multiplicative consistency property of membership degrees, $\mu_{i j}$, as considered by Liao and Xu in [13] is not sufficient to model the concept of consistency because it does neglect the multiplicative consistency property of non-membership degrees, $\nu_{i j}$. Therefore, the important question of correctly modelling the concept of consistency for IFPRs needs to be answered. In the following, a novel transitivity property for IFPRs is established by combining the multiplication operator of IFSs and Tanino's multiplicative transitivity property for FPRs [19]:

$$
p_{i j} \cdot p_{j k} \cdot p_{k i}=p_{i k} \cdot p_{k j} \cdot p_{j i}, \forall i, k, j .
$$

Extending the multiplicative transitivity of FPRs to the case of IFPRs would result in the following:

Definition 4 (Multiplicative Transitive IFPR). An IFPR $R=\left(r_{i j}\right)=\left(\left\langle\mu_{i j}, \nu_{i j}\right\rangle\right)$ on a finite set of alternatives $X$ is multiplicative transitive if and only if

$$
r_{i j} \otimes r_{j k} \otimes r_{k i}=r_{i k} \otimes r_{k j} \otimes r_{j i}, \quad \forall i, k, j .
$$

According to the operational laws of IFNs, the multiplicative transitivity property of IFPRs can be expressed as: $\mu_{i j} \mu_{j k} \mu_{k i}=$ $\mu_{i k} \mu_{k j} \mu_{j i}$ and $\nu_{i j}+\nu_{j k}-\nu_{i j} \nu_{j k}+\nu_{k i}-\left(\nu_{i j}+\nu_{j k}-\nu_{i j} \nu_{j k}\right) \nu_{k i}=\nu_{i k}+\nu_{k j}-\nu_{i k} \nu_{j k}+\nu_{k i}-\left(\nu_{i k}+\nu_{k j}-\nu_{i k} \nu_{k j}\right) \nu_{i k}$, which can be rewritten as $\mu_{i j} \mu_{j k} \mu_{k i}=\mu_{i k} \mu_{k j} \mu_{j i}$ and $\left(1-\nu_{i j}\right)\left(1-\nu_{j k}\right)\left(1-\nu_{k i}\right)=\left(1-\nu_{i k}\right)\left(1-\nu_{k j}\right)\left(1-\nu_{j i}\right)$. This is summarised in the following result: 
Proposition 1. An IFPR $R=\left(r_{i j}\right)=\left(\left\langle\mu_{i j}, \nu_{i j}\right\rangle\right)$ on a finite set of alternatives $X$ is multiplicative transitive if and only if $\forall i, k, j$ :

$$
\left.\begin{array}{l}
\mu_{i j} \mu_{j k} \mu_{k i}=\mu_{i k} \mu_{k j} \mu_{j i} \\
\left(1-\nu_{i j}\right)\left(1-\nu_{j k}\right)\left(1-\nu_{k i}\right)=\left(1-\nu_{i k}\right)\left(1-\nu_{k j}\right)\left(1-\nu_{j i}\right)
\end{array}\right\}
$$

Corollary 1. When an IFPR $R$ is reduced to a FPR $P$, (5) is equivalent to (3).

According to Proposition 1, the multiplicative transitivity property for an IFPR in Definition 4 can be rewritten as:

Definition 5 (Multiplicative Transitivity Property of IFPR). An IFPR $R$ on a finite set of alternatives $X$ is multiplicative transitive if and only if $\forall i, k, j$ :

$$
\left.\begin{array}{l}
\frac{\mu_{i j}}{\mu_{j i}}=\frac{\mu_{i k}}{\mu_{k i}} \cdot \frac{\mu_{k j}}{\mu_{j k}} \\
\frac{\left(1-v_{i j}\right)}{\left(1-v_{j i}\right)}=\frac{\left(1-v_{i k}\right)}{\left(1-v_{k i}\right)} \cdot \frac{\left(1-v_{k j}\right)}{\left(1-v_{j k}\right)}
\end{array}\right\}
$$

The multiplicative transitivity relationship in Definition 5 as represented in Fig. 1 verifies the following multiplicative consistency propagation rule:

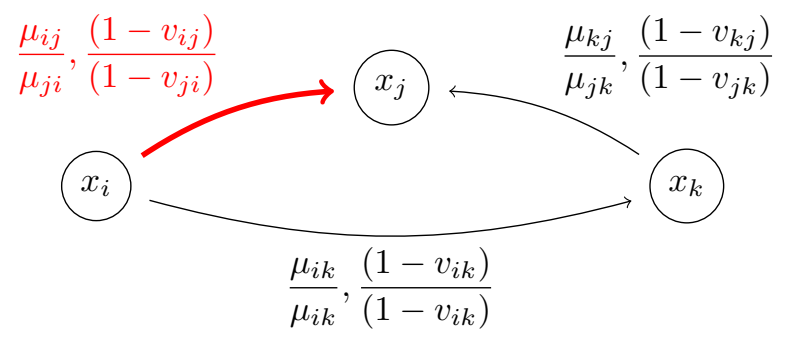

Fig. 1. The multiplicative transitivity of IFPRs between $x_{i}$ and $x_{j}$ via $x_{k}$

Proposition 2. If an IFPR $R$ is multiplicative transitive, then we have the following relationship:

$$
\frac{\mu_{i j}}{\mu_{j i}} \frac{\left(1-v_{i j}\right)}{\left(1-v_{j i}\right)}=\frac{\mu_{i k}}{\mu_{k i}} \frac{\left(1-v_{i k}\right)}{\left(1-v_{k i}\right)} \cdot \frac{\mu_{k j}}{\mu_{j k}} \frac{\left(1-v_{k j}\right)}{\left(1-v_{j k}\right)}, \forall i, k, j .
$$

Notice that the proposed multiplicative transitivity property for IFPRs given in Definition 5 includes Liao and Xu's definition of the same property given in [13], but it also includes an additional original foundation of multiplicative transitivity of IFPRs: $\left(1-\nu_{i j}\right)\left(1-\nu_{j k}\right)\left(1-\nu_{k i}\right)=\left(1-\nu_{i k}\right)\left(1-\nu_{k j}\right)\left(1-\nu_{j i}\right)$. Moreover, these original foundations are obtained by a strict mathematical derivation. Therefore, the proposed multiplicative transitivity in this article is reasonable and more appropriate to model the consistency of IFPRs than the proposed one in [13]. This reasonableness will further be strengthened by the multiplicative transitivity isomorphism between IFPRs and IVFPRs as the following section proves.

\section{Multiplicative Transitivity IsOMorPhism BetweEn IFPRS AND IVFPRS}

\section{A. Multiplicative Transitivity Property for IVFPRs}

In what follows, Zadeh's Extension Principle and the Representation Theorem of fuzzy sets [28] are applied to preference values that are fuzzy sets [32], which will then be characterised for the interval-valued fuzzy sets type, i.e. fuzzy sets whose membership function is always 1 for all values in a closed interval and zero otherwise, and consequently the multiplicative transitivity property for IVFPRs will be formally derived. First, the definition of an IVFPR is provided.

Definition 6. Let $\tilde{P}([0,1])$ be the set of closed intervals contained in the unit interval $[0,1]$. An IVFPR $B$ on a finite set of alternatives $X=\left\{x_{1}, \ldots, x_{n}\right\}$ is characterised by a membership function $\mu_{B}: X \times X \longrightarrow \tilde{P}([0,1])$ with $\mu_{B}\left(x_{i}, x_{j}\right)=$ $\widetilde{b}_{i j}=\left[b_{i j}^{-}, b_{i j}^{+}\right]$verifying $b_{i j}^{-}+b_{j i}^{+}=b_{i j}^{+}+b_{j i}^{-}=1, b_{i i}^{+}=b_{i i}^{-}=0.5 \quad \forall i, j=1,2, \ldots n$. Using matrix representation: $B=\left(\widetilde{b}_{i j}\right)=\left(\left[b_{i j}^{-}, b_{i j}^{+}\right]\right)$.

To extend the domain of a functional mapping from crisp elements to fuzzy sets the extension principle is applied [33]:

Definition 7. Let $X_{1} \times X_{2} \times \ldots \times X_{n}$ be a universal product set and $F$ be a functional mapping of the form $F: X_{1} \times$ $X_{2} \times \ldots \times X_{n} \longrightarrow Y$ that maps the element $\left(x_{1}, x_{2}, \ldots, x_{n}\right) \in X_{1} \times X_{2} \times \ldots \times X_{n}$ to the element $y=F\left(x_{1}, x_{2}, \ldots, x_{n}\right)$ of the universal set $Y$. Let $A_{i}$ be a fuzzy set over the universal set $X_{i}$ with membership function $\mu_{A_{i}}(i=1,2, \ldots, n)$. The membership function $\mu_{B}$ of the fuzzy set $B=F\left(A_{1}, \ldots, A_{n}\right)$ over the universal set $Y$ is

$$
\mu_{B}(y)=\sup _{y=F\left(x_{1}, \ldots, x_{n}\right)} \mu_{A_{1}}\left(x_{1}\right) * \ldots * \mu_{A_{n}}\left(x_{n}\right)
$$


When $\nexists\left(x_{1}, \ldots, x_{n}\right): y=F\left(x_{1}, \ldots, x_{n}\right)$ then $\mu_{B}(y)=0$, where $*$ is a t-norm.

In this article, the minimum t-norm $(\wedge)$ is used. Extending (3) to fuzzy sets requires the extension of $f:[0,1] \times[0,1] \times$ $[0,1] \longrightarrow[0,1]$ with $f\left(x_{1}, x_{2}, x_{3}\right)=x_{1} \cdot x_{2} \cdot x_{3}$. to $B=f\left(A_{1}, A_{2}, A_{3}\right)$ with $A_{1}, A_{2}, A_{3}$ fuzzy sets over [0,1] with membership functions $\mu_{A_{1}}, \mu_{A_{2}}, \mu_{A_{3}}$, respectively. According to Definition 7 , fuzzy set $B$ membership function is $\mu_{B}(y)=$ $\sup _{x_{1} \cdot x_{2} \cdot x_{3}=y} \mu_{A_{1}}\left(x_{1}\right) \wedge \mu_{A_{2}}\left(x_{2}\right) \wedge \mu_{A_{3}}\left(x_{3}\right)$. $x_{1} \cdot x_{2} \cdot x_{3}=y$
$x_{1}, x_{2}, x_{3} \in[0,1]$

A fuzzy set can also be represented horizontally via its corresponding family of crisp $\alpha$-level sets, in what it is known as the representation theorem of fuzzy sets. On the one hand, the $\alpha$-level set of fuzzy set $B$ is $B^{\alpha}=\left\{y \in[0,1] \mid \mu_{B}(y) \geq \alpha\right\}$, and $B=\bigcup_{0<\alpha \leq 1} \alpha B^{\alpha}$, with membership function $\mu_{B}(y)=\sup _{\alpha: y \in B_{\alpha}} \alpha$. On the other hand, if $A_{1}^{\alpha}, A_{2}^{\alpha}$ and $A_{3}^{\alpha}$ are the $\alpha$ level sets of $A_{1}, A_{2}$ and $A_{3}$ then $f\left(A_{1}^{\alpha} \times A_{2}^{\alpha} \times A_{3}^{\alpha}\right)=\left\{x_{1} \cdot x_{2} \cdot x_{3} \mid x_{1} \in A_{1}^{\alpha}, x_{2} \in A_{2}^{\alpha}, x_{3} \in A_{3}^{\alpha}\right\}$. Clearly, both $B^{\alpha}$ and $f\left(A_{1}^{\alpha} \times A_{2}^{\alpha} \times A_{3}^{\alpha}\right)$ are crisp sets. Furthermore, the following result holds:

Proposition 3. $B^{\alpha}=f\left(A_{1}^{\alpha} \times A_{2}^{\alpha} \times A_{3}^{\alpha}\right)$.

Proof. I. $f\left(A_{1}^{\alpha} \times A_{2}^{\alpha} \times A_{3}^{\alpha}\right) \subseteq B^{\alpha}$. Let $y \in f\left(A_{1}^{\alpha} \times A_{2}^{\alpha} \times A_{3}^{\alpha}\right)$. There exist $x_{1} \in A_{1}^{\alpha}, x_{2} \in A_{2}^{\alpha}$ and $x_{3} \in A_{3}^{\alpha}$ such that $x_{1} \cdot x_{2} \cdot x_{3}=y$. It is $\mu_{A_{1}}\left(x_{1}\right) \geq \alpha, \mu_{A_{2}}\left(x_{2}\right) \geq \alpha$ and $\mu_{A_{3}}\left(x_{3}\right) \geq \alpha$, which implies $\sup _{x_{1} \cdot x_{2} \cdot x_{3}=y} \mu_{A_{1}}\left(x_{1}\right) \wedge \mu_{A_{2}}\left(x_{2}\right) \wedge$ $x_{1} \in A_{1}^{\alpha}, x_{2} \in A_{2}^{\alpha}, x_{3} \in A_{3}^{\alpha}$

$\mu_{A_{3}}\left(x_{3}\right) \geq \alpha$. Because $A_{1}^{\alpha}, A_{2}^{\alpha}, A_{3}^{\alpha} \subseteq[0,1]$, then: $\sup _{\substack{x_{1} \cdot x_{2} \cdot x_{3}=y \\ x_{1}, x_{2}, x_{3} \in[0,1]}} \mu_{A_{1}}\left(x_{1}\right) \wedge \mu_{A_{2}}\left(x_{2}\right) \wedge \mu_{A_{3}}\left(x_{3}\right) \geq \sup _{\substack{x_{1} \cdot x_{2} \cdot x_{3}=y \\ x_{1} \in A_{1}^{\alpha}, x_{2} \in A_{2}^{\alpha}, x_{3} \in A_{3}^{\alpha}}} \mu_{A_{1}}\left(x_{1}\right) \wedge$ $\mu_{A_{2}}\left(x_{2}\right) \wedge \mu_{A_{3}}\left(x_{3}\right)$. It is concluded that $y \in B^{\alpha}$.

II. $B^{\alpha} \subseteq f\left(A_{1}^{\alpha} \times A_{2}^{\alpha} \times A_{3}^{\alpha}\right)$. Let $y \in B^{\alpha}$. By definition, it is $\mu_{B}(y) \geq \alpha$ and there exists at least three values $x_{1}, x_{2}, x_{3} \in$ $[0,1]$ such that $x_{1} \cdot x_{2} \cdot x_{3}=y$ and $\left[\mu_{A_{1}}\left(x_{1}\right) \wedge \mu_{A_{2}}\left(x_{2}\right) \wedge \mu_{A_{3}}\left(x_{3}\right)\right] \geq \alpha$. Therefore, it is true that $\mu_{A_{1}}\left(x_{1}\right) \geq \alpha, \mu_{A_{2}}\left(x_{2}\right) \geq$ $\alpha$ and $\mu_{A_{3}}\left(x_{3}\right) \geq \alpha$, which means that $x_{1} \in A_{1}^{\alpha}, x_{2} \in A_{2}^{\alpha}$ and $x_{3} \in A_{3}^{\alpha}$. Consequently, $y \in f\left(A_{1}^{\alpha} \times A_{2}^{\alpha} \times A_{3}^{\alpha}\right)$.

When $A_{1}, A_{2}$ and $A_{3}$ are interval-valued fuzzy sets with membership functions $\mu_{A_{i}}\left(x_{i}\right)=\left\{\begin{array}{ll}1 & x_{i} \in\left[a_{i}^{-}, a_{i}^{+}\right] \\ 0 & \text { Otherwise }\end{array}(i=1,2,3)\right.$, the only non-empty $\alpha$-level set of interval-valued fuzzy sets is the 1-level set, and therefore the application of Proposition 3 results in $f\left(A_{1}, A_{2}, A_{3}\right)=B=\underset{0<\alpha \leq 1}{\cup} \alpha B^{\alpha}=B^{1}=f\left(A_{1}^{1} \times A_{2}^{1} \times A_{3}^{1}\right)$. Interval arithmetic yields: $f\left(A_{1}^{1} \times A_{2}^{1} \times A_{3}^{1}\right)=$ $\left[a_{1}^{-} \cdot a_{2}^{-} \cdot a_{3}^{-}, a_{1}^{+} \cdot a_{2}^{+} \cdot a_{3}^{+}\right]$. Consequently, the multiplicative consistency property for IVFPRs can be defined as follows:

Definition 8 (Multiplicative Transitive IVFPR). An IVFPR $B=\left(\widetilde{b}_{i j}\right)=\left(\left[b_{i j}^{-}, b_{i j}^{+}\right]\right)$is multiplicative transitive if and only if

$$
\forall i, k, j:\left\{\begin{array}{l}
\frac{b_{i j}^{-}}{b_{j i}^{-}}=\frac{b_{i k}^{-}}{b_{k i}^{-}} \cdot \frac{b_{k j}^{-}}{b_{j k}^{-}} \\
\frac{b_{i j}^{+}}{b_{j i}^{+}}=\frac{b_{i k}^{+}}{b_{k i}^{+}} \cdot \frac{b_{k j}^{+}}{b_{j k}^{+}}
\end{array}\right.
$$

The multiplicative transitivity relationship in Definition 8 as represented in Fig. 2 verifies the following multiplicative consistency propagation rule:

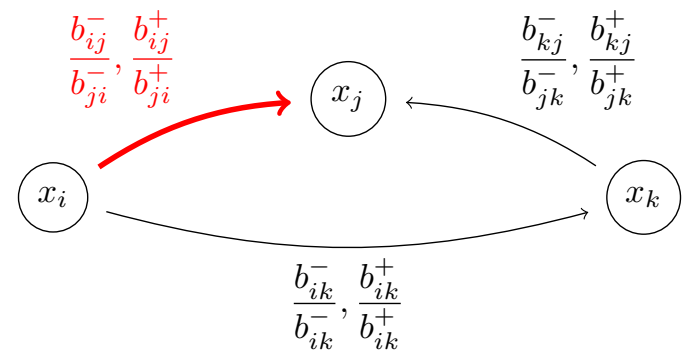

Fig. 2. The multiplicative transitivity of IVFPRs between $x_{i}$ and $x_{j}$ via $x_{k}$

Proposition 4. If an IVFPR $B=\left(\widetilde{b}_{i j}\right)=\left(\left[b_{i j}^{-}, b_{i j}^{+}\right]\right)$is multiplicative transitive, then we have the following relationship:

$$
\frac{b_{i j}^{-}}{b_{j i}^{-}} \frac{b_{i j}^{+}}{b_{j i}^{+}}=\frac{b_{i k}^{-}}{b_{k i}^{-}} \frac{b_{i k}^{+}}{b_{k i}^{+}} \cdot \frac{b_{k j}^{-}}{b_{j k}^{-}} \frac{b_{k j}^{+}}{b_{j k}^{+}}, \forall i, k, j .
$$




\section{B. Multiplicative Transitivity Isomorphism between IFPRs and IVFPRs}

Notice that given an IFS $A$, it is always true that $\mu_{A}(x) \leq 1-\nu_{A}(x)$. Since IFSs and interval-valued FSs (IVFSs) are mathematically equivalent [26], $\left[\mu_{A}(x), 1-\nu_{A}(x)\right]$ can be referred to as an intuitionistic fuzzy number (IFN) [30]. Therefore, an IFS can be seen as a collection of IFNs. Thus, the IFPR $R=\left(r_{i j}\right)=\left(\left\langle\mu_{i j}, \nu_{i j}\right\rangle\right)$ is mathematical isomorphic to the IVFPR $B=\left(\widetilde{b}_{i j}\right)=\left(\left[\mu_{i j}, 1-\nu_{i j}\right]\right)$ [27]. In what follows, it is proved that there also exists an isomorphism between the multiplicative transitivity properties for IFPRs and IVFPRs.

Theorem 1. Let $\mathcal{R}$ be the set of IFPRs and $\mathcal{B}$ be the set of IVFPRs. There exists a multiplicative transitivity isomorphism between $\mathcal{R}$ and $\mathcal{B}$.

Proof. Let $R=\left(r_{i j}\right)=\left(\left\langle\mu_{i j}, \nu_{i j}\right\rangle\right) \in \mathcal{R}$ be an IFPR and $B=\left(\widetilde{b}_{i j}\right)=\left(\left[b_{i j}^{-}, b_{i j}^{+}\right]\right)=\left(\left[\mu_{i j}, 1-\nu_{i j}\right]\right) \in \mathcal{B}$ be the isomorphic IVFPR. The following equivalence holds:

$$
\left.\begin{array}{l}
\frac{\mu_{i j}}{\mu_{j i}}=\frac{\mu_{i k}}{\mu_{k i}} \cdot \frac{\mu_{k j}}{\mu_{j k}} \\
\frac{\left(1-v_{i j}\right)}{\left(1-v_{j i}\right)}=\frac{\left(1-v_{i k}\right)}{\left(1-v_{k i}\right)} \cdot \frac{\left(1-v_{k j}\right)}{\left(1-v_{j k}\right)}
\end{array}\right\} \Leftrightarrow\left\{\begin{array}{l}
\frac{b_{i j}^{-}}{b_{j i}^{-}}=\frac{b_{i k}^{-}}{b_{k i}^{-}} \cdot \frac{b_{k j}^{-}}{b_{j k}^{-}} \\
\frac{b_{i j}^{+}}{b_{j i}^{+}}=\frac{b_{i k}^{+}}{b_{k i}^{+}} \cdot \frac{b_{k j}^{+}}{b_{j k}^{+}}
\end{array}\right.
$$

Thus, the IFPR $R$ is multiplicative transitive if and only if its isomorphic IVFPR $B$ is multiplicative transitive.

Theorem 1 reveals that IFPRs and IVFPRs do not only have a mathematical isomorphism, but also have a multiplicative transitivity isomorphism. The later is a substantial relationship between IFPRs and IVFPRs not reported up to now in literature. Furthermore, it gives a strong support for the reasonableness of the multiplicative transitivity properties for IFPRs and IVFPRs, which are constructed independently via different methodological approaches.

\section{Priority Vector Multiplicative Transitivity based MOP Model}

IVFPRs are suitable to deal with uncertainty derived when an expert may not possess a precise or sufficient level of knowledge of the problem to solve, while IFPRs are considered advantageous to express more comprehensive and intuitive decision making information than IVFPRs by making use of three tuple values coming from the variables: membership, non-membership and hesitation. In addition, if the hesitation degree is equal to zero, then IFPRs are reduced to FPRs. However, the derivation of the priority vector of IFPRs is more challenging than that of IVFPRs because of the more complex expression of the transitivity property of their mentioned three tuple values. Thus, a widely used approach to derive the priority vector of IFPRs is to convert them into their isomorphic IVFPRs, and derive the corresponding priority vector from the IVFPRs. However, to assure the validity of this approach, both the mathematical and multiplicative transitivity isomorphisms between IVFPRs and IFPRs are needed.

The isomorphism between IFPRs and IVFPRs derived in Section III implies that in the process of deriving priority vectors both preference representation formats can be regarded completely equivalent. Because interval-valued numbers arithmetics is less complex than intuitionistic fuzzy arithmetics, the derivation of the priority vector for an IFPR becomes a simpler task if it is converted into its equivalent IVFPR. Therefore, we only need use the isomorphic multiplicative transitivity property to model multiplicative consistency of IVFPRs and develop an appropriate multi-objective programming (MOP) model to derive its priority vector.

\section{A. Multiplicative Consistency of IVFPRs}

As aforementioned, the multiplicative transitivity property has been characterised as the most appropriate property for modelling consistency of FPRs [11], and because FPRs are particular cases of IVFPRs, this claim can be extended to this last representation format. The following result links the interval-valued priority weighting vector and the multiplicative transitivity property of an IVFPR associated to a set of alternatives.

Theorem 2. Let $W=\left(\widetilde{w}_{1}, \widetilde{w}_{2}, \ldots, \widetilde{w}_{n}\right)^{T}$ be the vector of interval-valued priority weights, $\widetilde{w}_{i}=\left[w_{i}^{-}, w_{i}^{+}\right]$associated to a set of alternatives $X=\left\{x_{1}, \ldots, x_{n}\right\}$. If

$$
\widetilde{b}_{i j}=\frac{\widetilde{w}_{i}}{\widetilde{w}_{i}+\widetilde{w}_{j}}=\left[\frac{w_{i}^{-}}{w_{i}^{+}+w_{j}^{+}}, \frac{w_{i}^{+}}{w_{i}^{-}+w_{j}^{-}}\right]
$$

then the IVFPR $B=\left(\widetilde{b}_{i j}\right)$ is multiplicative transitive.

Proof. Obvious.

Notice that weight vectors $c \cdot W$ with $c \in \mathbb{R}^{+}$also verify Theorem 2 and, following Tanino's result in [19], the following definition of multiplicative consistent IVFPR is justified: 
Definition 9. An IVFPR $B=\left(\widetilde{b}_{i j}\right)=\left(\left[b_{i j}^{-}, b_{i j}^{+}\right]\right)$is multiplicative consistent if and only if there exists a vector $W=$ $\left(\widetilde{w}_{1}, \widetilde{w}_{2}, \ldots, \widetilde{w}_{n}\right)^{T}, \widetilde{w}_{i}=\left[w_{i}^{-}, w_{i}^{+}\right]$, such that

$$
\forall i, j: \quad \widetilde{b}_{i j}=\frac{\widetilde{w}_{i}}{\widetilde{w}_{i}+\widetilde{w}_{j}}=\left[\frac{w_{i}^{-}}{w_{i}^{+}+w_{j}^{+}}, \frac{w_{i}^{+}}{w_{i}^{-}+w_{j}^{-}}\right]
$$

$W$ is unique up to multiplication by a positive scalar.

The multiplicative consistent IVFPR $B$ also verifies the following desirable properties:

Definition 10. An IVFPR $B=\left(\widetilde{b}_{i j}\right)=\left(\left[b_{i j}^{-}, b_{i j}^{+}\right]\right)$satisfies weak-transitivity if $b_{i j}^{-} \geq 0.5, b_{j k}^{-} \geq 0.5 \Rightarrow\left[b_{i k}^{-} \geq 0.5 \vee b_{i k}^{+} \geq 0.5\right]$.

Proposition 5. A multiplicative consistent IVFPR $B=\left(\widetilde{b}_{i j}\right)=\left(\left[b_{i j}^{-}, b_{i j}^{+}\right]\right)$satisfies weak-transitivity.

Proof. Because $b_{i j}^{-} \geq 0.5$, then $b_{j i}^{-}=1-b_{i j}^{+} \leq 1-b_{i j}^{-} \leq 1-0.5=0.5 ; \quad b_{j i}^{+}=1-b_{i j}^{-} \leq 0.5$. Thus,

$$
b_{j i}^{-} \leq b_{j i}^{+} \leq b_{i j}^{-} \leq b_{i j}^{+}
$$

Similarly, from $b_{j k}^{-} \geq 0.5$ it is

$$
b_{k j}^{-} \leq b_{k j}^{+} \leq b_{j k}^{-} \leq b_{j k}^{+}
$$

Using reductio ad absurdum, let us suppose that $b_{i k}^{-}<0.5$ and $b_{i k}^{+}<0.5$. In this case, it is $b_{i k}^{-}<0.5 \Rightarrow b_{k i}^{+}>0.5 ; b_{i k}^{+}<0.5 \Rightarrow$ $b_{k i}^{-}>0.5$ The following additional inequalities hold: $b_{k i}^{-}>b_{i k}^{-}, b_{k i}^{+}>b_{i k}^{+}$and because IVFPR $B$ is multiplicative consistent, applying (9) yields $b_{i j}^{-}=\frac{b_{k j}^{-} \cdot b_{i k}^{-}}{b_{j k}^{-} \cdot b_{k i}^{-}} \cdot b_{j i}^{-}<b_{j i}^{-}, b_{i j}^{+}=\frac{b_{i k}^{+} \cdot b_{k j}^{+}}{b_{j k}^{+} \cdot b_{k i}^{+}} \cdot b_{j i}^{+}<b_{j i}^{+}, b_{j k}^{-}=\frac{b_{j i}^{-} \cdot b_{i k}^{-}}{b_{i j}^{-} \cdot b_{k i}^{-}} \cdot b_{k j}^{-}<b_{k j}^{-}, b_{j k}^{+}=\frac{b_{j i}^{+} \cdot b_{k j}^{+}}{b_{i j}^{+} \cdot b_{k i}^{+}} \cdot b_{k j}^{+}<b_{k j}^{+} \cdot$ These inequalities contradict (13) and (14), and consequently it is true that $b_{i k}^{-} \geq 0.5 \vee b_{i k}^{+} \geq 0.5$.

Corollary 2. Given a multiplicative consistent $\operatorname{IVFPR} B=\left(\widetilde{b}_{i j}\right)=\left(\left[b_{i j}^{-}, b_{i j}^{+}\right]\right)$. The following condition holds:

$$
b_{i j}^{-} \geq 0.5, b_{j k}^{-} \geq 0.5 \Rightarrow b_{i k}^{+} \geq 0.5 \text {. }
$$

Proof. From Proposition 5, it is $b_{i k}^{-} \geq 0.5$ or $b_{i k}^{+} \geq 0.5$. If $b_{i k}^{-} \geq 0.5$ holds then it is also $b_{i k}^{+} \geq b_{i k}^{-} \geq 0.5$ and therefore $b_{i k}^{+} \geq 0.5$ holds in any case.

Proposition 5 implies that multiplicative consistency property for IVFPRs extends classical transitivity property of FPRs: when alternative $x_{i}$ is preferred to alternative $x_{j}$, and alternative $x_{j}$ is preferred to alternative $x_{k}$, then alternative $x_{i}$ is preferred to alternative $x_{k}$. Indeed, when the lower and upper preference bounds of an IVFPR are equal, Proposition 5 coincides with the weak-transitivity of FPRs.

Definition 11. An IVFPR $B=\left(\widetilde{b}_{i j}\right)=\left(\left[b_{i j}^{-}, b_{i j}^{+}\right]\right)$satisfies max - max-transitivity if $b_{i j}^{-} \geq 0.5, b_{j k}^{-} \geq 0.5 \Rightarrow\left[b_{i k}^{-} \geq b_{i j}^{-} \vee b_{i k}^{+} \geq\right.$ $\left.b_{i j}^{+}\right] \wedge\left[b_{i k}^{-} \geq b_{j k}^{-} \vee b_{i k}^{+} \geq b_{j k}^{+}\right]$.

Corollary 3. If an IVFPR $B=\left(\widetilde{b}_{i j}\right)=\left(\left[b_{i j}^{-}, b_{i j}^{+}\right]\right)$satisfies max-max-transitivity then it also satisfies weak-transitivity.

Proof. Obvious.

Proposition 6. A multiplicative consistent IVFPR $B=\left(\widetilde{b}_{i j}\right)=\left(\left[b_{i j}^{-}, b_{i j}^{+}\right]\right)$satisfies max - max-transitivity.

Proof. From Proposition 5, inequalities (13) and (14) hold. Using reductio ad absurdum:

- $b_{i k}^{-}<b_{j k}^{-} \wedge b_{i k}^{+}<b_{j k}^{+}$. Thus, it is $b_{i k}^{-}<b_{j k}^{-} \Rightarrow b_{k j}^{+}<b_{k i}^{+}$and $b_{i k}^{+}<b_{j k}^{+} \Rightarrow b_{k j}^{-}<b_{k i}^{-}$. Since IVFPR B is multiplicative consistent, then by Eq (9) it is concluded that $b_{i j}^{-}=\frac{b_{k j}^{-} \cdot b_{i k}^{-}}{b_{j k}^{-} \cdot b_{k i}^{-}} \cdot b_{j i}^{-}<b_{j i}^{-}, \quad b_{i j}^{+}<b_{j i}^{+}$. Obviously, this conclusion contradicts (13). Then, it is true that $b_{i k}^{-} \geq b_{j k}^{-} \vee b_{i k}^{+} \geq b_{j k}^{+}$.

- $b_{i k}^{-} \geq b_{j k}^{-} \wedge b_{i k}^{+} \geq b_{j k}^{+}$. Similarly, (14) would be contradicted and therefore it should be $b_{i k}^{-} \geq b_{j k}^{-} \vee b_{i k}^{+} \geq b_{j k}^{+}$.

Consequently, $\left[b_{i k}^{-} \geq b_{i j}^{-} \vee b_{i k}^{+} \geq b_{i j}^{+}\right] \wedge\left[b_{i k}^{-} \geq b_{j k}^{-} \vee b_{i k}^{+} \geq b_{j k}^{+}\right]$.

Corollary 4. Given a multiplicative consistent IVFPR $B=\left(\widetilde{b}_{i j}\right)=\left(\left[b_{i j}^{-}, b_{i j}^{+}\right]\right)$. The following condition holds:

$$
b_{i j}^{-} \geq 0.5, b_{j k}^{-} \geq 0.5 \Rightarrow b_{i k}^{+} \geq \max \left\{b_{i j}^{-}, b_{j k}^{-}\right\} .
$$

Proposition 6 implies that multiplicative consistency for IVFPRs extend max - max-transitivity property (also referred to as restricted - max-transitivity) of FPRs. Indeed, when the lower and upper preference bounds of an IVFPR are equal, Proposition 6 coincides with $\max -\max$-transitivity of FPRs, i.e. $b_{i k} \geq \max \left\{b_{i j}, b_{j k}\right\}$.

Definition 12. An IVFPR $B=\left(\widetilde{b}_{i j}\right)=\left(\left[b_{i j}^{-}, b_{i j}^{+}\right]\right)$satisfies the center - division-transitivity if

- $\forall i, j, k: b_{i j}^{-} \geq \lambda, b_{j k}^{-} \geq \lambda, \lambda \geq 0.5 \Rightarrow b_{i k}^{+} \geq \lambda$ 
- $\forall i, j, k: b_{i j}^{+} \leq \lambda, b_{j k}^{+} \leq \lambda, \lambda<0.5 \Rightarrow b_{i k}^{-} \leq \lambda$

Proposition 7. A multiplicative consistent IVFPR $B=\left(\widetilde{b}_{i j}\right)=\left(\left[b_{i j}^{-}, b_{i j}^{+}\right]\right)$satisfies center - division-transitivity.

Proof. The first statement derives from Corollary 4. Indeed, under the conditions $b_{i j}^{-} \geq \lambda, b_{j k}^{-} \geq \lambda, \lambda \geq 0.5$, it is true that $b_{i j}^{-} \geq 0.5, b_{j k}^{-} \geq 0.5$ and, consequently, it is $b_{i k}^{+} \geq \max \left\{b_{i j}^{-}, b_{j k}^{-}\right\}$and therefore it is $b_{i k}^{+} \geq \lambda$.

Assuming that $b_{i j}^{+} \leq \lambda, b_{j k}^{+} \leq \lambda, \lambda<0.5$, and using reductio ad absurdum it is also assumed that $b_{i k}^{-}>\lambda$, which implies $b_{i k}^{+} \geq b_{i k}^{-}>\lambda$. The following inequalities hold: $b_{i j}^{-} \leq b_{i j}^{+} \leq \lambda ; b_{j k}^{-} \leq b_{j k}^{+} \leq \lambda<1-\lambda \leq 1-b_{j k}^{+}=b_{k j}^{-} ; b_{k i}^{-}=1-b_{i k}^{+}<$ $1-\lambda \leq 1-b_{i j}^{+}=b_{j i}^{-}$. Because IVFPR B is multiplicative consistent, then by Eq (9) it is concluded that $b_{i k}^{-}=\frac{b_{i j}^{-} \cdot b_{j k}^{-} \cdot b_{k i}^{-}}{b_{k j}^{-} \cdot b_{j i}^{-}}<\lambda$. This contradicts the above assumption $b_{i k}^{-}>\lambda$, and therefore it is true $b_{i k}^{-} \leq \lambda$.

In Proposition 7, when $0.5 \leq \lambda \leq 1$, if the alternative $x_{i}$ is preferred over alternative $x_{j}$ with a maximum degree of preference of at least equal to $\lambda$, and alternative $x_{j}$ is preferred over alternative $x_{k}$ with a maximum degree of preference of at least equal to $\lambda$, then the maximum degree of preference of alternative $x_{i}$ over alternative $x_{k}$ will be at least equal to $\lambda$. However, if the maximum degrees of preferences above are at most equal to $\lambda \leq 0.5$, then the minimum degree of preference of alternative $x_{i}$ over alternative $x_{k}$ will not exceed $\lambda$.

\section{B. Multi-objective Programming Model for Deriving the Priority Vector of IVFPR}

In what follows, a multi-objective programming (MOP) methodology driven by the multiplicative consistency property is proposed to derive the priority vector of an IVFPR. To do this, first, the following notation is introduced:

$$
\eta_{i j}^{k}=\frac{b_{i k}^{-} b_{k j}^{-}}{b_{j k}^{-} b_{k i}^{-}} ; \quad \phi_{i j}^{k}=\frac{b_{i k}^{+} b_{k j}^{+}}{b_{j k}^{+} b_{k i}^{+}} .
$$

Given a multiplicative consistent IVFPR $B$, Definition 9 assures that there exists a vector $W=\left(\widetilde{w}_{1}, \widetilde{w}_{2}, \ldots, \widetilde{w}_{n}\right)^{T}, \widetilde{w}_{i}=$ $\left[w_{i}^{-}, w_{i}^{+}\right]$, which is unique up to multiplication by a positive scalar, such that $b_{i j}^{-}=\frac{w_{i}^{-}}{w_{i}^{+}+w_{j}^{+}}$and $b_{i j}^{+}=\frac{w_{i}^{+}}{w_{i}^{-}+w_{j}^{-}}$. In this case, it is also true that $\frac{b_{i j}^{-}}{1-b_{i j}^{+}}=\frac{b_{i j}^{-}}{b_{j i}^{-}}=\frac{b_{i k}^{-} b_{k j}^{-}}{b_{j k}^{-} b_{k i}^{-}}=\eta_{i j}^{k}$ and $\frac{b_{i j}^{+}}{1-b_{i j}^{-}}=\frac{b_{i j}^{+}}{b_{j i}^{+}}=\frac{b_{i k}^{+} b_{k j}^{+}}{b_{j k}^{+} b_{k i}^{+}}=\phi_{i j}^{k}$. Therefore, $b_{i j}^{-}=\frac{\eta_{i j}^{k}-\eta_{i j}^{k} \phi_{i j}^{k}}{1-\eta_{i j}^{k} \phi_{i j}^{k}}$ and $b_{i j}^{+}=\frac{\phi_{i j}^{k}-\eta_{i j}^{k} \phi_{i j}^{k}}{1-\eta_{i j}^{k} \phi_{i j}^{k}}$. Summarising:

Proposition 8. Let $B=\left(\widetilde{b}_{i j}\right)=\left(\left[b_{i j}^{-}, b_{i j}^{+}\right]\right)$be a multiplicative consistent IVFPR, and $W=\left(\widetilde{w}_{1}, \widetilde{w}_{2}, \ldots, \widetilde{w}_{n}\right)^{T}$ be its associated priority vector, where $\widetilde{w}_{i}=\left[w_{i}^{-}, w_{i}^{+}\right]$, then it is

$$
b_{i j}^{-}=\frac{w_{i}^{-}}{w_{i}^{+}+w_{j}^{+}}=\frac{\eta_{i j}^{k}-\eta_{i j}^{k} \phi_{i j}^{k}}{1-\eta_{i j}^{k} \phi_{i j}^{k}} \forall i, j, k
$$

and

$$
b_{i j}^{+}=\frac{w_{i}^{+}}{w_{i}^{-}+w_{j}^{-}}=\frac{\phi_{i j}^{k}-\eta_{i j}^{k} \phi_{i j}^{k}}{1-\eta_{i j}^{k} \phi_{i j}^{k}} \forall i, j, k
$$

Given an IVIFPR $B$, not necessarily multiplicative consistent, the interval-value $\widetilde{b}_{i j}^{k}=\left[b_{i j}^{k-}, b_{i j}^{k+}\right]=\left[\frac{\eta_{i j}^{k}-\eta_{i j}^{k} \phi_{i j}^{k}}{1-\eta_{i j}^{k} \phi_{i j}^{k}}, \frac{\phi_{i j}^{k}-\eta_{i j}^{k} \phi_{i j}^{k}}{1-\eta_{i j}^{k} \phi_{i j}^{k}}\right]$ is considered the partial multiplicative consistency estimation of the interval-value preference of alternative $x_{i}$ over alternative $x_{j}, b_{i j}$, obtained using the intermediate alternative $x_{k}$. The multiplicative consistency characterisation for FPRs derived by Chiclana et al. in [11] allows to just focus on the set of indexes $\{i, j, k \in\{1, \ldots, n\} \mid i<k<j\}$. The following deviation variables are always null when the IVFPR $B$ is multiplicative consistent:

$$
\left\{\begin{array}{c}
\varepsilon_{i j}^{k}=\left|w_{i}^{-}-b_{i j}^{k-} \cdot\left(w_{i}^{+}+w_{j}^{+}\right)\right| \\
\gamma_{i j}^{k}=\left|w_{i}^{+}-b_{i j}^{k+} \cdot\left(w_{i}^{-}+w_{j}^{-}\right)\right|
\end{array}\right.
$$

This is not the case when the IVFPR is not multiplicative consistent as (15) and (16) do not hold. In order to maximise multiplicative consistency, the priority vector for IVFPRs can be defined as the vector, $W=\left(\widetilde{w}_{1}, \widetilde{w}_{2}, \ldots, \widetilde{w}_{n}\right)^{T}$ with $\widetilde{w}_{i}=$ $\left[w_{i}^{-}, w_{i}^{+}\right]$, that minimises the sum of all the deviation variables $\varepsilon_{i j}^{k}$ and $\gamma_{i j}^{k}(i<k<j)$. Therefore, the following multi-objective programming (MOP1) problem can be established:

$$
\left\{\begin{aligned}
& \min J_{1}=\sum_{i=1}^{n} \sum_{j=i+2}^{n} \sum_{k=i+1}^{j-1} \varepsilon_{i j}^{k} \\
& \min J_{2}=\sum_{i=1}^{n} \sum_{j=i+2}^{n} \sum_{k=i+1}^{j-1} \gamma_{i j}^{k} \\
& \text { s.t. } 0 \leq w_{i}^{-} \leq w_{i}^{+} \leq 1 \\
& 0 \leq w_{j}^{-} \leq w_{j}^{+} \leq 1 \\
& i<k<j
\end{aligned}\right.
$$


For computing efficiency, the above MOP1 problem can be reduced to the following linear objective programming (LOP1) problem:

$$
\left\{\begin{aligned}
\min & J=\sum_{i=1}^{n} \sum_{j=i+2}^{n} \sum_{k=i+1}^{j-1}\left(\varepsilon_{i j}^{k}+\gamma_{i j}^{k}\right) \\
\text { s.t. } & 0 \leq w_{i}^{-} \leq w_{i}^{+} \leq 1 \\
0 & \leq w_{j}^{-} \leq w_{j}^{+} \leq 1 \\
i<k<j &
\end{aligned}\right.
$$

The solution of the above LOP1 problem is obtained by solving the linear objective programming (LOP2) problem:

$$
\left\{\begin{array}{l}
\min J=\sum_{i=1}^{n} \sum_{j=i+2}^{n} \sum_{k=i+1}^{j-1}\left[\left(\varepsilon_{i j}^{k-}+\varepsilon_{i j}^{k+}\right)+\left(\gamma_{i j}^{k-}+\gamma_{i j}^{k+}\right)\right] \\
\text { s.t. } w_{i}^{-}-\frac{\eta_{i j}^{k}-\eta_{i j}^{k} \phi_{i j}^{k}}{1-\eta_{i j}^{k} \phi_{i j}^{k}}\left(w_{i}^{+}+w_{j}^{+}\right)-\varepsilon_{i j}^{k-}+\varepsilon_{i j}^{k+}=0 \\
w_{i}^{+}-\frac{\phi_{i j}^{k}-\eta_{i j}^{k} \phi_{i j}^{k}}{1-\eta_{i j}^{k} \phi_{i j}^{k}}\left(w_{i}^{-}+w_{j}^{-}\right)-\gamma_{i j}^{k-}+\gamma_{i j}^{k+}=0 \\
0 \leq w_{i}^{-} \leq w_{i}^{+} \leq 1 \\
0 \leq w_{j}^{-} \leq w_{j}^{+} \leq 1 \\
\varepsilon_{i j}^{k-} \geq 0, \varepsilon_{i j}^{k+} \geq 0, \gamma_{i j}^{k-} \geq 0, \gamma_{i j}^{k+} \geq 0 \\
i<k<j
\end{array}\right.
$$

The following result holds:

Theorem 3. The IVFPR $B=\left(\widetilde{b}_{i j}\right)=\left(\left[b_{i j}^{-}, b_{i j}^{+}\right]\right)$is multiplicative consistent if and only if $J^{*}=0$ where $J^{*}$ is the optimal value of the LOP2 problem.

Proof. (Sufficiency) If IVFPR $B=\left(\widetilde{b}_{i j}\right)=\left(\left[b_{i j}^{-}, b_{i j}^{+}\right]\right)$is multiplicative consistent, then the deviation variables $\varepsilon_{i j}^{k}$ and $\gamma_{i j}^{k}$ are all equal to zero, and therefore $J^{*}=0$.

(Necessity) If $J^{*}=0$, then $\sum_{i=1}^{n} \sum_{j=1}^{n} \sum_{k=1}^{m}\left[\left(\varepsilon_{i j}^{k-}+\varepsilon_{i j}^{k+}\right)+\left(\gamma_{i j}^{k-}+\gamma_{i j}^{k+}\right)\right]=0$. Because $\varepsilon_{i j}^{k-} \geq 0, \varepsilon_{i j}^{k+} \geq 0, \gamma_{i j}^{k-} \geq 0$ and $\gamma_{i j}^{k+} \geq 0$ $\forall i<k<j$, then it is $\varepsilon_{i j}^{k-}=\varepsilon_{i j}^{k+}=\gamma_{i j}^{k-}=\gamma_{i j}^{k+}=0 \quad \forall i<k<j$. This implies that: $b_{i j}^{-}=\frac{w_{i}^{-}}{w_{i}^{+}+w_{j}^{+}}$and $b_{i j}^{+}=\frac{w_{i}^{+}}{w_{i}^{-}+w_{j}^{-}} \forall i<j$. Applying reciprocity property of $B$, the above will also be true for all $j<i$. Thus, Definition 9 is verified and $B$ is multiplicative consistent.

\section{NumericAl EXAMPLE}

With stronger public awareness and increasing government regulation in environmental protection, green supplier selection has become an important issue of operation for all companies. However, the decision making process for green supplier selection is complex because it relates to: (1) quantitative criteria (price, quality, delivery deadline, etc); and (2) qualitative criteria (environment management, pollution control, etc). The former is easily dealt with crisp numbers, while the later is suitable to be expressed by IFSs due to the lack of knowledge.

An electric automobile company aims to select the most appropriate green supplier for one of the key elements in its manufacturing process. After pre-evaluation by qualitative criteria, four suppliers $A_{i}(i=1,2,3)$ have remained as alternatives for further evaluation. For simplicity, just one qualitative criteria is considered: environment management. The expert provides his/her preference relation on $\left\{A_{1}, A_{2}, A_{3}, A_{4}\right\}$ with the following IFPR, $R$ :

$$
\left(\begin{array}{cccc}
\langle 0.50,0.50\rangle & \langle 0.35,0.55\rangle & \langle 0.50,0.30\rangle & \langle 0.40,0.50\rangle \\
\langle 0.55,0.35\rangle & \langle 0.50,0.50\rangle & \langle 0.60,0.20\rangle & \langle 0.20,0.40\rangle \\
\langle 0.30,0.50\rangle & \langle 0.20,0.60\rangle & \langle 0.50,0.50\rangle & \langle 0.40,0.40\rangle \\
\langle 0.50,0.40\rangle & \langle 0.40,0.20\rangle & \langle 0.40,0.40\rangle & \langle 0.50,0.50\rangle
\end{array}\right) .
$$

The isomorphic IVFPRs, $B$, is:

$$
\left(\begin{array}{llll}
{[0.50,0.50]} & {[0.35,0.45]} & {[0.50,0.70]} & {[0.40,0.50]} \\
{[0.55,0.65]} & {[0.50,0.50]} & {[0.60,0.80]} & {[0.20,0.60]} \\
{[0.30,0.50]} & {[0.20,0.40]} & {[0.50,0.50]} & {[0.40,0.60]} \\
{[0.50,0.60]} & {[0.40,0.80]} & {[0.40,0.60]} & {[0.50,0.50]}
\end{array}\right)
$$

The associated LOP2 problem derives the following priority vector is (using MATLAB) $\left[w_{1}^{-}, w_{1}^{+}\right]=[0.053,0.068] ;\left[w_{2}^{-}, w_{2}^{+}\right]=$ $[0.221,0.238] ; \quad\left[w_{3}^{-}, w_{3}^{+}\right]=[0.038,0.062] ; \quad\left[w_{4}^{-}, w_{4}^{+}\right]=[0.078,0.141]$. 
Applying the possibility degree (PD) to measure the ordering relation between two interval-valued numbers [35], $P\left(a_{1} \succ\right.$ $\left.a_{2}\right)=\max \left\{1-\max \left\{\frac{a_{2}^{+}-a_{1}^{-}}{a_{1}^{+}-a_{1}^{-}+a_{2}^{+}-a_{2}^{-}}, 0\right\}, 0\right\}$, results in the following possibility degree $(P D)$ matrix corresponding to the above interval priority vector components:

$$
P D=\left(\begin{array}{cccc}
0.50 & 0.00 & 0.77 & 0.00 \\
1.00 & 0.50 & 1.00 & 1.00 \\
0.23 & 0.00 & 0.50 & 0.00 \\
1.00 & 0.00 & 1.00 & 0.50
\end{array}\right)
$$

Using the arithmetic mean operator the following crisp priority vector is obtained: $w_{1}=0.3175 ; w_{2}=0.8750 ; w_{3}=$ $0.1825 ; w_{4}=0.6250$. Thus, the final ranking of alternatives is $A_{2} \succ A_{4} \succ A_{1} \succ A_{3}$ and the best green supplier is $A_{2}$.

The solution to the programming problem that derives from Using Model (3) in Liao and Xu [13] has the objective function value $Z^{*}=0.6157$ and the optimal priority vectort $\left[w_{1}^{-}, w_{1}^{+}\right]=[0.192,0.192] ; \quad\left[w_{2}^{-}, w_{2}^{+}\right]=[0.301,0.410] ; \quad\left[w_{3}^{-}, w_{3}^{+}\right]=$ $[0.115,0.268] ; \quad\left[w_{4}^{-}, w_{4}^{+}\right]=[0.240,0.335]$. Using the above resolution process, the possibility degree $\left(P D_{L X}\right)$ matrix corresponding to the interval priority vector components would be now:

$$
P D_{L X}=\left(\begin{array}{cccc}
0.500 & 0.000 & 0.503 & 0.000 \\
1.000 & 0.500 & 1.000 & 1.000 \\
0.497 & 0.000 & 0.50 & 0.113 \\
1.000 & 0.000 & 0.887 & 0.500
\end{array}\right)
$$

The arithmetic mean operator would produce now the following crisp priority vector: $w_{1}=0.2508 ; w_{2}=0.8750 ; w_{3}=$ $0.2775 ; w_{4}=0.5968$. Thus, the ranking order of alternatives is $A_{2} \succ A_{4} \succ A_{3} \succ A_{1}$ and the best green supplier is $A_{2}$. Notice that the proposed MOP methodology in this article produces the same best alternative as Liao and Xu's method, although there is a difference in the reversal ordering of alternatives $A_{1}$ and $A_{3}$. Also, the priority vector component associated to $A_{1}$ by Liao and $\mathrm{Xu}$ is a crisp number: $\left[w_{1}^{-}, w_{1}^{+}\right]=0.192$, which is unexpected because all input values are interval-valued. This information loss is due to the usage of the multiplicative transitivity property for the membership degrees but not the corresponding property for non-membership degrees, which is required as the latter cannot be derived from the former one.

The solution of the fractional programming Model 1 in Liao and $\mathrm{Xu}$ [36] has the objective function value $F^{*}=0.7$, and the optimal priority vector $\omega_{1}=0.2, \omega_{2}=0.3, \omega_{3}=0.2, \omega_{4}=0.3$. Then, the ranking order of alternatives is $A_{2} \sim A_{4} \succ A_{3} \sim A_{1}$. Therefore, in this decision making case, the consistency property of IFPRs proposed in [36] does not allow the proper order relation vector because its priority vector is a collection of crisp numbers. This crisp priority vector means that information is lost in the order ranking process of IFPRs. We believe that this is because the proposed consistency property of IFPRs in [36] is a straight forward extension of the respective proposal for the case of IVFPRs [37], and therefore it is not rich enough to capture all the information contained in IFNs: membership, non-membership and hesitation.

\section{Vi. Analysis of the Proposed Multiplicative Transitivity Property of IFPRs}

The consistency based models for IFPRs proposed in [14]-[16] are based on the application of the corresponding consistency property of FPRs to intuitionistic fuzzy preferences without providing a solid mathematical foundation for its validity, i.e. without taking into account the characteristics of the multiplication of intuitionistic fuzzy sets. Indeed, some of the consistency properties for IFPRs proposed in literature have subsequently been proved to be inappropriate to model consistency of IFPRs. This article overcomes this issue by providing the mathematical framework for the correct formulation of the multiplicative transitivity for IFPRs, and as such it presents the following main advantages with respect to the proposed models in [14]-[16]:

(1) This article investigates the isomorphic multiplicative transitivity property for IFPRs, which is based on the formal extension of the multiplicative consistency property for FPRs proposed Tanino [19]. The proposed multiplicative transitivity property for IFPRs in this article includes Liao and Xu's definition of the same property given in [13], $\mu_{i j} \mu_{j k} \mu_{k i}=\mu_{i k} \mu_{k j} \mu_{j i}$, but it also includes another additional original foundation of multiplicative transitivity of IFPRs, $\left(1-\nu_{i j}\right)\left(1-\nu_{j k}\right)(1-$ $\left.\nu_{k i}\right)=\left(1-\nu_{i k}\right)\left(1-\nu_{k j}\right)\left(1-\nu_{j i}\right)$. These two original foundations are obtained by following a strict mathematical derivation. Therefore, the proposed multiplicative transitivity in this article is reasonable and more appropriate to model the consistency of IFPRs than the proposed one in [13]. Furthermore, it proves that the priority vector derived by the isomorphic multiplicative transitivity proved in this article contains more information than the crisp priority vector derived using the approach proposed in [36].

(2) This article also proves that there exists a multiplicative transitivity isomorphism between IFPRs and IVFPRs. This isomorphism strengthens the reasonability of the derived multiplicative transitivity property for IFPRs because both types of multiplicative transitivity for IFPRs and IVFPRs are established via two different but robust mathematical approaches: Operational laws of IFSs and Extension Principle and Representation Theorem of FSs, respectively. Consequently, the relationship between IFPRs and IVFPRs is twofold: mathematical isomorphism (superficial) and multiplicative transitivity isomorphism (substantial). Thus, this relationship guarantees the conversion between IFPRs and IVFPRs to be effective in [27]. Furthermore, it fills the gap between hesitation (IFPRs) and uncertainty (IVFPRs) in MCDM problems. It is worth noting that this has not been yet reported in the literature on this topic. 
(3) This article defines the concept of multiplicative consistency for IFPRs based on the isomorphic multiplicative transitivity property, and it is proved to satisfies several desirable properties: weak-transitivity, max - max-transitivity and center - division-transitivity. The multiplicative consistency is also used to propose a multi-objective programming (MOP) methodology to derive the priority vector of IFPRs, which in turn is converted into a corresponding linear objective programming model for computing efficiency.

\section{CONCLUSIONS}

This article established the multiplicative transitivity property for IFPRs by combining the multiplication of IFSs and the extension of Tanino's multiplicative transitivity for FPRs. Then, it was proved that there exists a multiplicative transitivity isomorphism between IFPRs and IVFPRs, which strengthens its reasonability because it is established by using two different reliable methodologies, respectively. Therefore, IFPRs and IVFPRs are equal in twofold: (1) mathematical isomorphism (superficial); (2) multiplicative transitivity isomorphism (substantial), and hesitation and uncertainty can be converted into each other in MCDM problems. Furthermore, the multiplicative consistency of IVFPRs was proved to satisfy the following desirable properties: weak-transitivity, $\max$ - max-transitivity and center - division-transitivity. Finally, a multiplicative consistency based MOP methodology was proposed to derive the priority vector for IVFPRs and consequently for IFPRs, which is solved via the a corresponding linear objective programming model for computing efficiency. A discussion highlighting the main differences between the multiplicative transitivity property presented in this article and the existing ones is also included.

The proposed multiplicative transitivity assumes that IFPRs are complete. However, in some realistic decision making case, due to lack of knowledge or pressure of time, an expert would not be able to efficiently express any kind of preference degree between two or more of the available alternatives, and as a consequence they might provide incomplete information [38]-[40]. The multiplicative transitivity MOP approach for deriving the priority vector of incomplete IFPRs will be different form the one investigated in this article for complete IFPRs. Therefore, future research efforts are required to develop a multiplicative transitivity property based model to deal with missing elements in incomplete IFPRs and an appropriate method to derive their priority vector.

\section{ACKNOWLEDGMENT}

This work was supported by National Natural Science Foundation of China (71571166,71501135), Zhejiang Provincial Natural Science Foundation of China (LY15G010003), Zhejiang Provincial Philosophy and Social Science Foundation-Zhijiang Young Talent of Social Science (16ZJQN046YB), China Postdoctoral Science Foundation (2016T90863), the Scientific Research Foundation for Excellent Young Scholars at Sichuan University (2016SCU04A23).

\section{REFERENCES}

[1] H. Nurmi, (1981). Approaches to collective decision making with fuzzy preference relations. Fuzzy Sets and Systems 6, 249-259.

[2] M. M. Xia, Z. S. Xu and H. C. Liao, (2013). Preference relations based on intuitionistic multiplicative information. IEEE Transactions on Fuzzy Systems $21,113-133$.

[3] Z. S. Xu, (2013). Priority weight intervals derived from intuitionistic multiplicative preference relations. IEEE Transactions on Fuzzy Systems 21 , 642-654.

[4] K. T. Atanassov, (1986). Intuitionistic fuzzy sets. Fuzzy Sets and Systems 20, 87-96.

[5] Z. S. Xu and H. C. Liao, (2015). A survey of approaches to decision making with intuitionistic fuzzy preference relations. Knowledge-Based Systems 80 , 131-142.

[6] S. P. Wan, F. Wang, L. L. Lin and J. Y. Dong, (2015). An intuitionistic fuzzy linear programming method for logistics outsourcing provider selection. Knowledge-Based Systems $82,80-94$.

[7] S. P. Wan, F. Wang and J. Y. Dong, (2016). A novel group decision making method with intuitionistic fuzzy preference relations for RFID technology selection. Applied Soft Computing 36, 405-422.

[8] J. Wu, (2015). Consistency in MCGDM Problems with Intuitionistic Fuzzy Preference Relations Based on an Exponential Score Function. Group Decision and Negotiation 25, 399-420.

[9] S. Z. Zeng, W. H. Su and L. R. Sun, (2013). A method based on similarity measures for interactive group decision-making with intuitionistic fuzzy preference relations. Applied Mathematical Modelling 37, 6909-6917.

[10] J. Wu , R. Xiong and F. Chiclana, (2016). Uninorm trust propagation and aggregation methods for group decision making in social network with four tuples information. Knowledge-Based Systems 96, 29-39.

[11] F. Chiclana, E. Herrera-Viedma, S. Alonso and F. Herrera, (2009). Cardinal consistency of fuzzy preference relations: a characterization of multiplicative transitivity. IEEE Transactions on Fuzzy Systems 17, 14-23.

[12] Z. S. Xu, X. Q. Cai and E. Szmidt, (2011). Algorithms for estimating missing elements of incomplete intuitionistic preference relations. International Journal of Intelligent Systems 26, 787-813.

[13] H. C. Liao and Z. S. Xu, (2014). Priorities of intuitionistic fuzzy preference relation based on multiplicative consistency. IEEE Transactions on Fuzzy Systems 22, 1669-1681.

[14] Z. W. Gong, L. S. Li, F. X. Zhou and T. X. Yao, (2009). Goal programming approaches to obtain the priority vectors from the intuitionistic fuzzy preference relations. Computers and Industrial Engineering 57, 1187-1193.

[15] Z. W. Gong, L. S. Li, F. Jeffrey and Y. Zhao, (2011). The optimal priority models of the intuitionistic fuzzy preference relation and their application in selecting industries with higher meteorological sensitivity. Expert Systems with Applications 38, 4394-4402.

[16] Z. J. Wang, (2013). Derivation of intuitionistic fuzzy weights based on intuitionistic fuzzy preference relations. Applied Mathematical Modelling 37, 6377-6388.

[17] E. Herrera-Viedma, F. Herrera, F. Chiclana and M. Luque, (2004). Some issues on consistency of fuzzy preference relations. European Journal of operational Research 154, 98-109.

[18] A. K. Sen, (1977). Social choice theory: a re-examination. Econometrica, 45, 53-89. 
[19] T. Tanino, (1984). Fuzzy preference orderings in group decision making. Fuzzy Sets and system 12, $117-131$.

[20] E. Barrenechea, J. Fernandez, M. Pagola, F. Chiclana and H. Bustince, (2014). Construction of interval-valued fuzzy preference relations from ignorance functions and fuzzy preference relations. Knowledge-Based Systems 58, 33-44.

[21] F. Liu, W. G. Zhang and L, H. Zhang, (2014). A group decision making model based on a generalized ordered weighted geometric average operator with interval preference matrices. Fuzzy Sets and Systems 246, 1-18.

[22] Z. J. Wang and K. W. Li, (2015). A multi-step goal programming approach for group decision making with incomplete interval additive reciprocal comparison matrices. European Journal of Operational Research 242, 890-900.

[23] J. Wu and F. Chiclana, (2014). A social network analysis trust-consensus based approach to group decision-making problems with interval-valued fuzzy reciprocal preference relations. Knowledge-Based Systems 59, 97-107.

[24] Y. Dong, C.-C. Li, F. Chiclana and E. Herrera-Viedma, (2016) Average-case consistency measurement and analysis of interval-valued reciprocal preference relations. Knowledge-Based Systems doi: 10.1016/j.knosys.2016.10.005

[25] Y. J. Xu, K. W. Li and H. M. Wang, (2014). Incomplete interval fuzzy preference relations and their applications. Computers and Industrial Engineering 67, 93-103.

[26] G. Deschrijver and E. E. Kerre, (2003). On the relationship between some extensions of fuzzy set theory. Fuzzy Sets and Systems 133, 227-235.

[27] J. Wu and F. Chiclana, (2012). Non-dominance and attitudinal prioritisation methods for intuitionistic and interval-valued intuitionistic fuzzy preference relations. Expert Systems with Applications 39, 13409-13416.

[28] L. A. Zadeh, (1975). The concept of a linguistic variable and its application to approximate reasoning-I. Information Sciences 8, $199-249$.

[29] J. Wu and F. Chiclana, (2014). A risk attitudinal ranking method for interval-valued intuitionistic fuzzy numbers based on novel attitudinal expected score and accuracy functions. Applied Soft Computing 22, 272-286.

[30] Z. S. Xu and R. R. Yager, (2006). Some geometric aggregation operators based on intuitionistic fuzzy sets. International Journal of General Systems $35,417-433$.

[31] Z. S. Xu, (2007). Intuitionistic preference relations and their application in group decision making. Information Sciences 177, $2363-2379$.

[32] J. Wu and F. Chiclana, (2014). Multiplicative consistency of intuitionistic reciprocal preference relations and its application to missing values estimation and consensus building. Knowledge-Based Systems 71, 187-200.

[33] M. Hanss, (2005). Applied Fuzzy Arithmetic. An Introduction with Engineering Applications. Springer-Verlag Berlin Heidelberg.

[34] F. Chiclana, E. Herrera-Viedma, F. Herrera and S. Alonso, (2007). Some induced ordered weighted averaging operators and their use for solving group decision-making problems based on fuzzy preference relations. European Journal of Operational Research 182, 383-399.

[35] Z. S, Xu and Q. L. Da, (2002). The uncertain OWA operator. International Journal of Intelligent Systems 17, 569-575.

[36] H. C. Liao and Z. S, Xu, (2014). Automatic procedures for group decision making with intuitionistic preference relations. Journal of Intelligent and Fuzzy Systems 27, 2341-2353.

[37] Z. S, Xu, (2011). Consistency of interval fuzzy preference relations in group decision making. Applied Soft Computing 11, 3898-3909.

[38] S. Alonso, E. Herrera-Viedma, F. Chiclana and F. Herrera. (2010). A web based consensus support system for group decision making problems and incomplete preferences. Information Sciences 180, 4477-4495.

[39] R. Ureña, F. Chiclana, J. A. Morente-Molinera and E. Herrera-Viedma, (2015). Managing incomplete preference relations in decision making: A review and future trends. Information Sciences 302, 14-32.

[40] R. Ureña, F. Chiclana, H. Fujita and E. Herrera-Viedma, (2015). Confidence-consistency driven group decision making approach with incomplete reciprocal intuitionistic preference relations. Knowledge-Based Systems 89, 86-96.

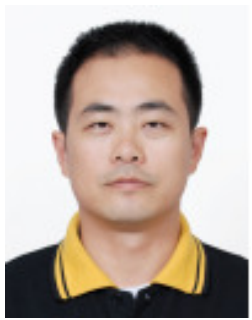

Professor Jian Wu received the $\mathrm{BSc}$ and $\mathrm{PhD}$ degrees in management science and engineering, both from Hefei University of Technology, China, in 2000 and 2008, respectively. Dr Jian Wu is currently a distinguished professor with the School of Economics and Management, Shanghai Maritime University, China. His research interests include fuzzy preference modelling, group decision making and information fusion. He has $30+$ papers published in leading international journals.

From October 2012 to October 2013, Dr Jian Wu was at De Montfort University as an academic research visitor in the Centre for Computational Intelligence (CCI). He is an Associate Editor of the Journal of Intelligent and Fuzzy Systems and Guest Editor of theApplied Soft Computing journal.

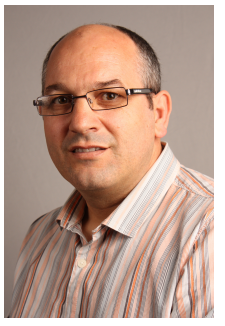

Professor Francisco Chiclana received the BSc and PhD degrees in Mathematics, both from the University of Granada (Spain) in 1989 and 2000, respectively. He is currently a Professor of Computational Intelligence and Decision Making at De Montfort University (Leicester, UK) and Honorary Professor in the Department of Mathematics (University of Leicester) from 1 July 2015 to 30 June 2018

Professor Chiclana is Associate Editor of IEEE Transactions on Systems, Man, and Cybernetics: Systems, Information Sciences, Soft Computing Journal, International Journal of Fuzzy Systems, and the Journal of Intelligent and Fuzzy Systems. He also serves as member of the editorial board of Applied Soft Computing, Knowledge-Based Systems, and Multiple-Valued Logic and Soft Computing. He has published extensively in leading international journals with over 70 journal articles indexed in Web of Science. Several of his papers (currently 15) are classed as highly cited by the Essential Science Indicators database from Thomson Reuters (ESI). He has also been consistently classed as one of the Most Cited Scientist in its field by ESI. His current research interests include decision making, computational intelligence, information fusion, consensus, rule induction and sentiment analysis. 


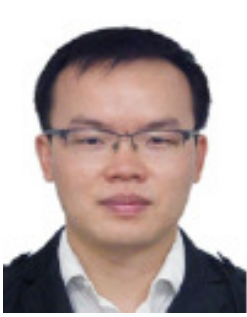

Huchang Liao is an Associate Research Fellow in the Business School at the Sichuan University, Chengdu, China. He received his $\mathrm{PhD}$ degree in Management Science and Engineering from the Shanghai Jiao Tong University, Shanghai, China, in 2015. From Oct 2013 to Oct 2014, he was an Academic Visitor with the School of Computer Science, University of Manchester, UK. From July 2015 to Dec 2015, he was an Endeavour Research Fellow at the University of Technology Sydney.

He has published 2 monographs and nearly 50 peer-reviewed papers in high-quality international journals. He currently has 13 papers classed as Highly Cited Papers and 3 of them as Hot Papers by ESI. His current research interest includes group decision making, computing with words, information fusion, and multiple criteria decision making under uncertainty.

Dr Liao is the Associate Editor of International Journal of Fuzzy Systems, Academic Editor of British Journal of Mathematics \& Computer Science and Editorial Board Member of some international journals. Dr. Liao research has been recognised with the 2015 Endeavour Research Fellowship granted by the Australia Government; the 2015 Outstanding Graduate of Shanghai by the Shanghai Government; the 2012, 2013, 2014 Chinese National Scholarship for PhD Students Awards; the 2013 Chinese State Scholarship Fund Award; the 2013 Outstanding PhD Thesis Foundation Award granted by the Shanghai Jiao Tong University. 